\title{
Wnt5a/FZD4 Mediates the Mechanical Stretch-Induced Osteogenic Differentiation of Bone Mesenchymal Stem Cells
}

\author{
Qingguo Gu Haijun Tian ${ }^{\mathrm{a}}$ Kai Zhang ${ }^{\mathrm{a}}$ Deyu Chen ${ }^{\mathrm{b}}$ Dechun Chen ${ }^{\mathrm{b}}$ \\ Xinwei Wang ${ }^{b}$ Jie Zhao \\ aShanghai Key Laboratory of Orthopaedic Surgery, Shanghai Ninth People's Hospital,Shanghai Jiao \\ Tong University School of Medicine, Shanghai, 'Department of Spine Surgery, Changzheng Hospital, \\ The Second Military Medical University, Shanghai, China
}

\section{Key Words}

Mechanical loading • BMSCs • FZD4 • Osteogenesis • Osteoporosis

\begin{abstract}
Background/Aims: Mechanical stimulation and WNT signalling have essential roles in regulating the osteogenic differentiation of bone marrow stromal cells (BMSCs) and bone formation. However, little is known regarding the regulation of WNT signalling molecule expression and therefore the osteogenic differentiation of BMSCs during osteogenesis. Methods: Microarrays of BMSCs from elderly individuals or patients with osteoporosis (GSE35959) from the GEO database were analysed using GeneSight-Lite 4.1.6 (BioDiscovery) and C2 curated gene sets downloaded from Molecular Signatures Database (MSigDB). Realtime PCR and western blotting were used to measure the expression of the indicated genes. ALP and Alizarin red staining were used to evaluate the osteogenesis of BMSCs. Results: In this study, we investigated whether mechanical loading directly regulates the expression of WNT signalling molecules and examined the role of WNT signalling in mechanical loadingtriggered osteogenic differentiation and bone formation. We first studied the microarrays of samples from patients with osteoporosis and found downregulation of the GPCR ligand binding gene set in the BMSCs of patients with osteoporosis. Then, we demonstrated that mechanical stimuli can regulate osteogenesis and bone formation both in vivo and in vitro. FZD4 was upregulated during cyclic mechanical stretch (CMS)-induced osteogenic differentiation, and the JNK signalling pathway was activated. FZD4 knockdown inhibited the mechanical stimuli-induced osteogenesis and JNK activity. More importantly, we found an activating effect of WNT5A and FZD4 that regulated bone formation in response to hindlimb unloading in mice, and pretreatment with WNT5A or activation of the expression of FZD4 partly rescued the osteoporosis caused by mechanical unloading. Conclusions: Our results demonstrate, for the first time, that mechanical stimulation alters the expression of genes involved in the osteogenic differentiation of BMSCs via the direct regulation of FZD4 and that therapeutic WNT5A and FZD saRNA may be an efficient strategy for enhancing bone formation under mechanical stimulation.




\section{Introduction}

Osteoporosis, one of the most common skeletal disorders in the elderly population, is currently attributed to impaired differentiation of bone marrow-derived mesenchymal stem cells (BMSCs), which are progenitors of osteoblasts and adipocytes in bone [1]. It has been demonstrated that in osteoporosis, BMSCs favour adipogenesis over osteogenesis [2-4]. Thus, uncovering the complex regulation pathways of normal BMSC differentiation and restoring the decreased osteogenic differentiation of BMSCs is a promising therapeutic strategy for osteoporosis. Decreases in mechanical loading in long-duration bed-rest patients may incur significant bone loss [5, 6], showing the importance of biophysical signals in regulating the differentiation of BMSCs. Mechanical stretching has previously been shown to be a fundamental regulator of BMSC differentiation in vitro. It has been found that cyclic mechanical tension can induce the osteogenesis of BMSCs and that mechanical compression induces chondrogenic differentiation [7, 8]. For example, $10 \%$ cyclic mechanical strain induces the expression of osteogenic marker genes, such as alkaline phosphatase (ALP) and Runx2, and enhances calcium deposition [9]. In addition, mechanical stretch can also increase the secretion of various growth or cytokine factors, such as bone morphogenetic protein 2 (BMP2) and therefore modulate bone remodelling and homeostasis. However, a deeper understanding of the mechanisms underlying the regulation of BMSC differentiation by mechanical cues is required.

In addition to physical signals, the fate of BMSCs is also influenced by chemical (including BMPs, Wnt, MAPKs and Notch) signals $[10,11]$. The importance of both canonical and noncanonical Wnt signalling in the osteogenesis of stem cells has been widely explored in many studies in vivo and in vitro $[12,13]$. Wnt5a signalling suppresses adipogenesis by suppressing the transcriptional activity of PPAR- $\gamma$ and promotes the differentiation of BMSCs into osteoblasts [14, 15]. Wnt5a knockout mice showed decreased bone formation with enhanced adipogenesis and impaired osteogenesis [16]. Wnt5a, a noncanonical Wnt ligand, activates signalling through both $\beta$-catenin-independent mediators, such as c-Jun N-terminal Kinase (JNK) and the $\beta$-catenin-dependent pathway [17]. Feng et al. found that activated JNK signalling promotes the osteogenic differentiation of mouse MC3T3-E1 cells [18]. Furthermore, previous studies have demonstrated that mechanical stress also enhanced the expression of Wnt5a/Wnt5b mRNA in stem cells and autonomously promoted osteogenic differentiation. Extracellular Wnt5 ligands bind Frizzled4 (FZD4) receptors (class FZD G-protein-coupled receptors (GPCRs)) at the cell membrane. However, there is little information about whether the expression of FZD4 would be regulated by mechanical stretch and whether FZD4 functions as the receptor of Wnt5a during osteogenesis.

In this study, we wondered whether FZD4 functions as the receptor of Wnt5a in the mechanical stretch-induced osteogenic differentiation of BMSCs. By analysing the microarray data from osteoporotic patients, we found decreased expression of FZD receptors in the BMSCs of osteoporotic patients. Mechanical stretch upregulated the expression of FZD4 and Wnt5a and therefore induced the osteogenesis of BMSCs in vitro. Knockdown of FZD4 by using a specific shRNA inhibited the mechanical stretch-induced osteogenic differentiation of BMSCs in vitro and bone formation in vivo. These data demonstrated that the FZD4 receptor mediated the osteogenic effect of Wnt5a in response to mechanical stimuli, and our results may provide new strategies to improve mechanical stretch-enhanced bone formation.

\section{Materials and Methods}

Cell culture, shRNA knockdown, and lentiviral transduction overexpression studies

BMSCs were flushed out with $\alpha$-minimum essential medium ( $\alpha$-MEM, Gibco) from the femurs of 10 - to 12-week-old mice and were then cultured in growth medium ( $\alpha$-MEM including $10 \%$ foetal bovine serum (FBS) (Gibco)) at $37{ }^{\circ} \mathrm{C}$ with 5\% CO2. The attached BMSCs were used for experiments at passages 3 to 7 . The pLenti-C-Myc-FZD4 shRNA vectors that targeted FZD4 and the empty control vectors (scram-shRNA) 


\section{Cellular Physiology Cell Physiol Biochem 2018;48:215-226

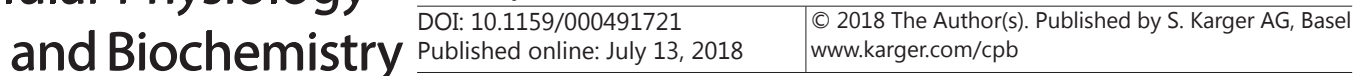

Gu et al.: Wnt5a Mediates Mechanical Stretch Induced Osteogenesis

were purchased from OriGene Technologies, Inc. The lentiviruses were packaged via a Lenti-vpak packaging kit (OriGene Technologies, Inc., Medical Center, Rockville) and then transfected into human BMSCs using FuGENE $^{\circledR}$ HD Transfection Reagent (Roche Applied Science, Mannheim, Germany). After incubation for forty-eight hours, the viral supernatant was collected and used for infection. The FZD4 gene was ligated into the pcDNA3.1myc-His vector (Invitrogen). The empty and pcDNA3.1myc-His-FZD4 constructs were transfected into viral packaging cells. After incubation for forty-eight hours, the supernatant was collected and used for the viral infection of BMSCs. Cells stably expressing GFP were gathered by sorting for GFPpositive cells using fluorescence-activated cell sorting.

\section{Microarray analysis and gene-set enrichment analysis (GSEA)}

We analysed the microarrays of BMSCs from elderly individuals or patients with osteoporosis (GSE35959) from the GEO database. After normalization of the expression profiles using GeneSight-Lite 4.1.6 (BioDiscovery), C2 curated gene sets downloaded from Molecular Signatures Database (MSigDB) were tested for enrichment based on the GSEA method [19]. Genes were sorted according to the value of the t-statistic computed against the human BMSC "Normal (control) vs. Osteoporosis" phenotype, with genes upregulated in the "control" group at the left-end of the list and genes upregulated in the "osteoporosis" group at the right-end. The heat map was partly explored for genes differentially expressed between the BMSCs of the two groups.

\section{Hindlimb unloading mouse model and bone histomorphometric analyses}

Six-month-old male C57BL/6J mice were purchased from Shanghai SLAC Laboratory Animal Co. Ltd. The hindlimbs were suspended for a period of 28 days, as previously described. The Committees of Animal Ethics and Experimental Safety of Shanghai Ninth People's Hospital approved all of the experimental procedures. We measured the structure of the tibial plateau with a SCANCO Medical $\mu \mathrm{CT} 40$ scanner. Then, images were analysed using SCANCO evaluation software to perform a three-dimensional morphometric analysis and determine the density and distance parameters (SCANCO Medical AG, Switzerland). The threedimensional structural parameters analysed included the following: BMD (bone mineral density), BV/ TV (bone volume/tissue volume), Tb.Th (trabecular thickness), Tb.Sp (trabecular separation), and SMI (structure model index). WNT5A was overexpressed in mice by injection of mouse recombinant WNT5A protein via the tail vein, and FZD4 was overexpressed in mice by the saRNA (small activating RNA) technique.

\section{Alkaline phosphatase staining and activity assay}

ALP staining was performed on cultured control and mechanically stretched cells. After being rinsed with PBS three times, the cell layer was fixed in 4\% paraformaldehyde for $15 \mathrm{~min}$ at room temperature. The cells were then incubated with buffer containing $0.1 \%$ naphthol AS-Bi phosphate (Sigma-Aldrich, St. Louis, MO, USA) and $2 \%$ fast violet B (Sigma-Aldrich, St. Louis, MO, USA). After incubation for $1 \mathrm{~h}$ at $37^{\circ} \mathrm{C}$, the cell layer was washed with deionized water. An ALP activity assay was performed at $405 \mathrm{~nm}$ using p-nitrophenyl phosphate (pNPP) (Sigma Aldrich) as the substrate. Briefly, a $50 \mathrm{ml}$ sample was incubated with $50 \mathrm{ml}$ of pNPP $(1 \mathrm{mg} / \mathrm{ml})$ in $1 \mathrm{M}$ diethanolamine buffer containing $0.5 \mathrm{mM} \mathrm{MgCl}_{2}(\mathrm{pH} 9.8)$ at $37{ }^{\circ} \mathrm{C}$ for $15 \mathrm{~min}$. The reaction was stopped by adding $2 \mathrm{M} \mathrm{NaOH}$ to $200 \mu \mathrm{l}$ of the reaction mixture. The total protein content was determined by the BCA method with a protein assay kit (PIERCE, Rockford, IL). ALP activity was determined as nmol p-nitrophenol per minute per mg protein and presented as the fold change relative to that of the control group.

\section{Alizarin red staining}

After being fixed in $70 \%$ ice-cold ethanol for $1 \mathrm{~h}$ and rinsed with double-distilled $\mathrm{H}_{2} \mathrm{O}$, the cells were then stained with $40 \mathrm{mM}$ Alizarin red S (pH 4.9, Sigma, St. Louis, MO, USA) for 15 min with gentle agitation. After staining, the cells were rinsed five times with double-distilled $\mathrm{H}_{2} \mathrm{O}$. For the quantitative assessment of the degree of mineralization, the red stain was eluted by $10 \%(\mathrm{w} / \mathrm{v})$ cetylpyridinium chloride (SigmaAldrich) for $1 \mathrm{~h}$ and quantified via spectrophotometric absorbance measurements of the OD at $570 \mathrm{~nm}$.

Cyclic mechanical stretch (CMS) application

Cells were cultured at a density of $2 \times 10^{5}$ cells $/ \mathrm{cm}^{2}$ in $1.5 \mathrm{ml}$ of medium on six-well flexible silicone rubber BioFlex ${ }^{\mathrm{TM}}$ plates coated with collagen type I (Flexcell International Corporation, Hillsborough, 


\section{Cellular Physiology Cell Physiol Biochem 2018;48:215-226 \begin{tabular}{c|c} 
DOI: 10.1159/000491721 & Ond Biochemistry \\
Published online: July 13, 2018 & $\begin{array}{l}\text { 2018 The Author(s). Published by S. Karger AG, Basel } \\
\text { www.karger.com/cpb }\end{array}$
\end{tabular}}

Gu et al.: Wnt5a Mediates Mechanical Stretch Induced Osteogenesis

NC). After being cultured for $24 \mathrm{~h}$ to reach $50-60 \%$ confluency, cyclic mechanical stretch (CMS, with a 0.5 $\mathrm{Hz}$ sinusoidal curve at $10 \%$ elongation) was applied to the cells. CMS was applied using an FX-5000T TM Flexercell ${ }^{\circledast}$ Tension Plus ${ }^{\mathrm{TM}}$ unit (Flexcell International Corporation) in a humidified atmosphere at $37^{\circ} \mathrm{C}$ and $5 \% \mathrm{CO}_{2}$, as previously reported [20]. Mechanically stretched cells were harvested immediately after the application of the CMS. In all of the following experiments, control cells were plated in the same plates but were not subjected to stretching.

\section{Quantitative real-time PCR ( $q$ RT-PCR)}

The total RNA of cells was isolated using TRIzol reagent (Invitrogen, Carlsbad, CA, USA) according to the manufacturer's instructions. First-strand cDNA was synthesized from $1 \mu \mathrm{g}$ of total RNA by incubation for $1 \mathrm{~h}$ at $42{ }^{\circ} \mathrm{C}$ with Superscript III reverse transcriptase (Invitrogen) following oligo(dT) priming. After the reverse transcription reaction, the real-time polymerase chain reaction (PCR) was performed with an ABI 7900HT system using SYBR ${ }^{\circledR}$ Premix (Takara, Dalian, China) according to the manufacturer's instructions. The conditions for real-time PCR were as follows: denaturation at $95^{\circ} \mathrm{C}$ for $10 \mathrm{~s}$ and 40 cycles of $95^{\circ} \mathrm{C}$ for $10 \mathrm{~s}$ and $60^{\circ} \mathrm{C}$ for $30 \mathrm{~s}$. A dissociation stage was added to the end of the amplification procedure. No nonspecific amplification was observed, as determined using the dissociation curve. Glyceraldehyde 3-phosphate dehydrogenase (GAPDH) was used as an internal control. The data were analysed using the comparison $\mathrm{Ct}\left(2^{-\Delta \Delta t}\right)$ method and expressed as the fold change relative to the respective control. Each sample was analysed in triplicate. The primer sequences used in this study were as follows: GAPDH: forward, 5'-AGGTCGGTGTGAACGGATTTG-3'; reverse, 5'-GGGGTCGTTGATGGCAACA-3'; ALP: forward, 5'-CGTCTCCATGGTGGATTATGC3'; reverse, 5'-TGGCAAAGACCGCCACAT-3'; Runx2: forward, 5'-GACTGTGGTTACCGTCATGGC-3'; reverse, 5'- ACTTGGTTTTTCATAACAGCGGA-3'; Col1a1: forward, 5'-GCTCCTCTTAGGGGCCACT-3'; reverse, 5'- ATTGGGGACCCTTAGGCCAT-3'; Wnt5a: forward, 5'- TCGACTATGGCTACCGCTTTG $\quad-3$ '; reverse, 5'-CACTCTCGTAGGAGCCCTTG-3'; Wht9a: forward, 5'- ATGCTGGATGGGTCCCTTCT-3'; reverse, 5'-ACTGCCTGTTAGCCCGAAGTA-3', FZD4: forward, 5'-CCTCGGCTACAACGTGACC-3'; reverse, 5'- TGCACATTGGCACATAAACAGA-3', FZD1: forward, 5'-CAGCAGTACAACGGCGAAC-3'; reverse, 5'- GTCCTCCTGATTCGTGTGGC -3', FZD6: forward, 5'-TCTGCCCCTCGTAAGAGGAC-3'; reverse, 5'- GGGAAGAACGTCATGTTGTAAGT-3', FZD7: forward, 5'- GTGCCAACGGCCTGATGTA-3'; reverse, 5'-AGGTGAGAACGGTAAAGAGCG-3', Rac1: forward, 5'-ATGTCCGTGCAAAGTGGTATC-3'; reverse, 5'- CTCGGATCGCTTCGTCAAACA-3', Ror1: forward, 5'- CAGTCAGTGCTGAATTAGTGCC-3'; reverse, 5'- TCATCGAGGGTCAGGTAAGAAT-3', Ror2: forward, 5'-GTGCGGTGGCTAAAGAATGAT-3'; reverse, 5'- ATTCGCAGTCGTGAACCATATT-3'.

\section{Western blot analysis}

Cells were collected from the BioFlex ${ }^{\mathrm{TM}}$ plates and were then lysed on ice for $30 \mathrm{~min}$ in lysis buffer containing $50 \mathrm{mM}$ Tris- $\mathrm{HCl}(\mathrm{pH} 7.4), 150 \mathrm{mM} \mathrm{NaCl}, 1 \%$ Nonidet P-40, and $0.1 \%$ SDS supplemented with protease inhibitors (10 mg/ml leupeptin, $10 \mathrm{mg} / \mathrm{ml}$ pepstatin $\mathrm{A}$, and $10 \mathrm{mg} / \mathrm{ml}$ aprotinin). Protein fractions were collected by centrifugation at $15,000 \mathrm{~g}$ at $4{ }^{\circ} \mathrm{C}$ for $10 \mathrm{~min}$. Protein concentrations were determined using the Micro Bicinchoninic acid (BCA) Assay Kit (Thermo Fisher Scientific). In addition, 20 $\mu \mathrm{g}$ of total protein extract was then subjected to $10 \%$ or $12 \%$ sodium dodecyl sulfate-polyacrylamide gel electrophoresis (SDS-PAGE) and transferred to nitrocellulose membranes (Whatman, Piscataway, NJ). After being blocked with 5\% BSA, the membranes were incubated with according antibodies overnight at $4{ }^{\circ} \mathrm{C}$. We used the following primary antibodies to determine the concentrations of proteins in the samples: antiRunx2 mAb (1:1000, M-70, Santa Cruz Biotech, Santa Cruz Biotechnology), anti-P-JNK1, anti-total-JNK1 mAb (1:1000; ab199380, Abcam, Cambridge, UK), anti-Wnt5a mAb (1:1000, ab72583, Abcam, Cambridge, UK), anti-FZD4 mAb (1:1500, Cell Signalling Technology, Inc.), and anti-GAPDH mAb (1:5000, Sigma-Aldrich). A horseradish peroxidase-conjugated secondary antibody was added and visualized using an enhanced chemiluminescence detection system (Millipore, Billerica, MA) as recommended by the manufacturer.

\section{Histological and histomorphometric analysis}

For the assessment of new bone formation, we injected green fluorescent calcein (Sigma; $5 \mathrm{mg} / \mathrm{kg}$ body weight) and red fluorescent Alizarin red (Sigma, $10 \mathrm{mg} / \mathrm{kg}$ ) into the mice on days 7 and 2 before euthanasia. Left tibias were fixed in 4\% paraformaldehyde in PBS (pH 7.4) for $24 \mathrm{~h}$, subsequently decalcified in $10 \%$ EDTA for ca. 1 month, and then embedded in paraffin. Histological sections $(7 \mu \mathrm{m}$ thick) were prepared 
Gu et al.: Wnt5a Mediates Mechanical Stretch Induced Osteogenesis

for TRAP with haematoxylin counterstain. The specimens were then examined and photographed using a high quality microscope. The percentage of osteoclast surface per bone surface (OcS/BS, \%) was assessed for each sample. Bone histomorphometric analyses of the mineral apposition rate (MAR) were performed using professional image analysis software (ImageJ; NIH, Bethesda, MD, USA) and fluorescence microscopy (Leica, Q500MC). The bone histomorphometric parameters were calculated and expressed according to the standardized nomenclature for bone histomorphometry.

Statistical analysis

The data are presented as the mean \pm S.D. ( $n$ is the number of tissue preparations, cells or experimental replicates). For comparing groups of data, a two-tailed Student's $t$-test was used. A value of $\mathrm{P}<0.05$ was considered statistically significant.

\section{Results}

Bone formation and FZD4 expression are impaired in the BMSCs of osteoporotic patients and HU mice

The expression profiles of BMSCs from osteoporotic and age-matched normal people were analysed (The microarrays were downloaded from the GEO database, GEO accession number GSE35958) using the GSEA method. In a previous report, the skeletal development gene set was enriched in the BMSCs from the normal people compared to that of the osteoporotic group [20]. In the present study, we found that the GPCR ligand binding gene set (which contains Wnts and receptors, such as FZD1, FZD4, FZD6 and FZD7) was also obviously enriched within the sorted list (the NES score was -2.162 and the FDR value for the gene set was 0.002) (Fig. 1a). Genes that were differentially expressed by at least 2-fold in this set were highlighted in a heat map (Fig. 1b). The receptors of Wnts, such as FZD1, FZD4, FZD6 and FZD7, were downregulated in the osteoporosis group. The expression of genes coding noncanonical Wnts, such as Wnt5a and Wnt9a, were also decreased in the osteoporosis group (Fig. 1b). To investigate whether the inhibited osteogenic differentiation of the MSCs of osteoporotic patients was regulated by the expression of Wnt5a and FZDs, we used a hindlimb unloading mouse model and performed bone histomorphometric analyses by measuring the bone density by micro-computed tomography (microCT) (Fig. 1c). A significant decrease in BMD, BV/TV, and Tb.Th was observed, indicating the bone loss was caused by the mechanical unloading (Fig. 1d).

Then, we isolated the BMSCs from normal and hindlimb unloading (HU) mice to study the expression of Wnt5a and FZD4 and the osteogenic differentiation ability. It was shown that osteogenic differentiation was significantly impaired by mechanical unloading, as assessed by the ALP staining (Fig. 1e) and Alizarin red staining of the in vitro mineralization in BMSCs (Fig. 1f). Consistently, quantitative real-time PCR (qRT-PCR) analysis showed that the expression of the osteogenic marker genes Col1a1, Alp and Runx2 was also obviously downregulated in the HU group (Fig. $1 \mathrm{~g}$ ). The mRNA expression levels of Wnt5a, Wnt9a and FZD4 were found to be downregulated in the HU BMSCs (Fig. 1h), in accordance with the microarray results of osteoporotic patients. Thus, this finding reminded us that the impaired osteogenic differentiation of MSCs in osteoporotic patients or HU mice might be partly because of the decreased expression of Wnt5a and FZD4. We also need to investigate whether the decreased expression of Wnt5a and FZD4 resulted from mechanical unloading and the underlying mechanisms.

Cyclic mechanical stretch promotes the expression of Wnt5a/FZD4 and the osteogenic differentiation of BMSCs

To investigate how mechanical stretch regulates osteogenic differentiation in vitro, we used a CMS-induced osteogenic differentiation model of human BMSCs. After loading for 96 $\mathrm{h}, \mathrm{qRT}-\mathrm{PCR}$ analysis showed that the expression of the osteogenic marker genes Alp, Runx2, and Col1a1 was higher in the CMS group than in the no-loading control cells (Ctrl group) 
Fig. 1. Bone formation and FZD4 expression are impaired in the BMSCs of osteoporotic patients and HU mice. (a) GSEA of the expression profiles of BMSCs from normal (control) (average donor age $57.6 \pm 9.56$ years, four female) and osteoporotic (average donor age $86.2 \pm 5.89$ years, four female) patients. Enrichment curves computed by GSEA are shown in green (FDR-corrected $\mathrm{P}<0.05$ ). GSEA for the GPCR ligand binding gene set demonstrated significant enrichment in control human BMSCs compared with BMSCs from osteoporotic patients. (b) The

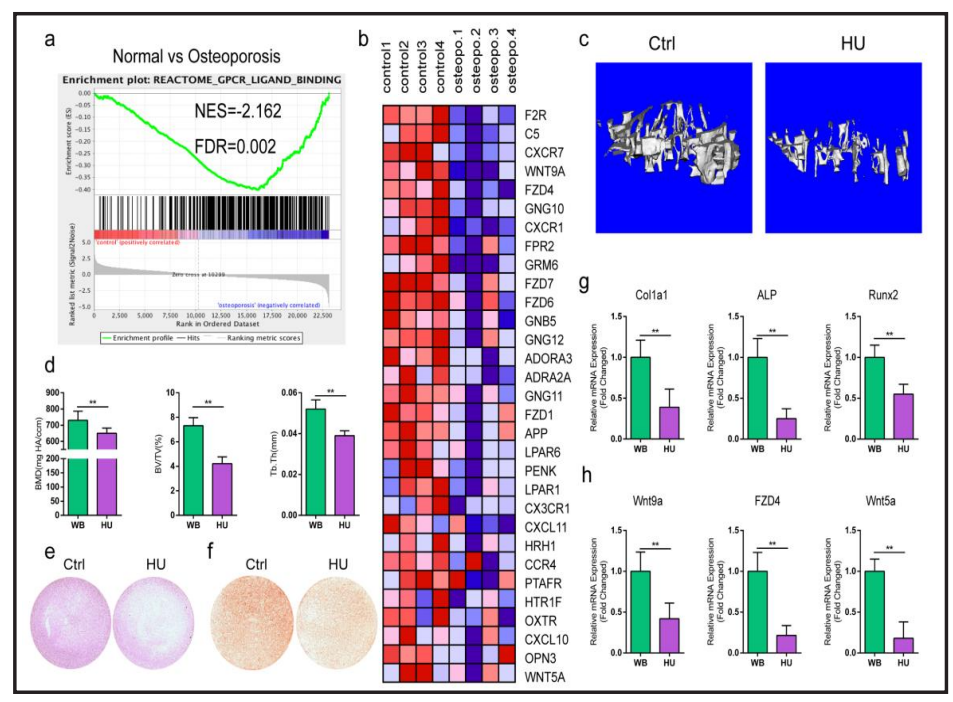
heat map is ordered by the degree of differential expression of GPCR ligand binding genes between the BMSCs from normal (control) and osteoporotic patients. (c) Representative microCT reconstruction images of the tibial plateau of WB and HU mice. $n=6$. (d) Three-dimensional microstructural parameters of the tibial plateau of WB and HU mice. (e) ALP activity of BMSCs isolated from WB and HU mice after 7 days of osteogenic induction were determined with alkaline phosphatase staining. (f) Mineralized nodules formed by BMSCs isolated from WB and HU mice after 14 days of osteogenic induction were determined with Alizarin Red staining. (g) Relative Col1a1, ALP and Runx2 mRNA expression in BMSCs isolated from WB and HU mice. (h) Relative Wnt9a, FZD4 and wnt5a mRNA expression in BMSCs isolated from WB and HU mice. All the data were confirmed in three repeated tests. Data are the mean \pm S.D. ${ }^{* *} \mathrm{P}<0.01$. All P-values are based on Student's t-test.

(Fig. 2a), which was confirmed by the results of the western blot analysis of Runx2 (Fig. 2b). Consistent with the above changes, $10 \%$ CMS treatment also enhanced Alizarin red staining (Fig. 2c), as confirmed by quantitative analysis. These results showed that CMS promoted the osteogenic differentiation of BMSCs. Moreover, CMS also increased the mRNA and protein expression of Wnt5a (Fig. 2d, 2e), a previously reported mechanical cue-induced osteogenic factor. The expression levels of Wnt5a receptors, Frizzled (FZDs), Rac1, the tyrosine kinaselike orphan receptors Ror1, and Ror2 were all examined. The result showed that only the mRNA expression of FZD4, FZD6, Ror1 and Ror2 was upregulated under CMS stimulation, during which FZD4 expression was the most increased (Fig. 2f). We further confirmed this result by FZD 4 protein expression examination (Fig. 2g). To confirm the fundamental role of FZD4 in mediating the osteogenic effect of Wnt5a, we designed specific siRNAs for the abovementioned receptors, and we found that FZD4 siRNA most obviously decreased the expression of osteogenic marker Runx2 under the condition of Wnt5a stimulation (Fig. 2h). Then, we focused on the effect of FZD4 in the CMS-induced osteogenic differentiation of BMSCs.

\section{FZD4 is indispensable for the CMS-induced osteogenic differentiation of BMSCs}

Next, we used RNA interference to knockdown FZD4 to confirm that FZD4 is indispensable for the CMS-induced osteogenic differentiation of BMSCs. Compared to scrambled shRNA, the FZD4-specific shRNA significantly decreased the expression of FZD4, as shown by RT-PCR and western blot analyses (Fig. 3a and 3b). We found that CMS activated the JNK signalling pathway, shown as an increased expression of phosphorylated JNK and an unchanged total JNK protein level. CMS also upregulated the protein expression of Runx2. However, these effects were significantly blocked by FZD4 shRNA (Fig. 3c). The CMS-induced osteogenic differentiation of BMSCs, shown by the ALP staining, was also decreased after 
Fig. 2. Cyclic mechanical stretch promotes the expression of Wnt5a/FZD4 and the osteogenic differentiation of BMSCs. (a) The ALP, Col1a1 and Runx2 mRNA expression was determined by quantitative real-time PCR (qRT-PCR) with or without the application of CMS for $48 \mathrm{~h}, 72 \mathrm{~h}$ and $96 \mathrm{~h}$. (b) Western blot analysis the Runx2 protein expression after the application of CMS for $48 \mathrm{~h}$, $72 \mathrm{~h}$ and $96 \mathrm{~h}$. GAPDH was used as the internal control. (c) The mineralized nodules formed in BMSCs after the application of CMS for 14 days compared with those in control cells were evaluated with Alizarin Red staining and quantified. (d) The Wnt5a mRNA expression was determined by quantitative real-time PCR (qRTPCR) after treatment with or without CMS for $48 \mathrm{~h}, 72 \mathrm{~h}$ and $96 \mathrm{~h}$. (e) Western blot analysis the Wnt5a protein expression after the application of CMS for $48 \mathrm{~h}$, $72 \mathrm{~h}$ and $96 \mathrm{~h}$. GAPDH was used as the internal control.(f) The mRNA

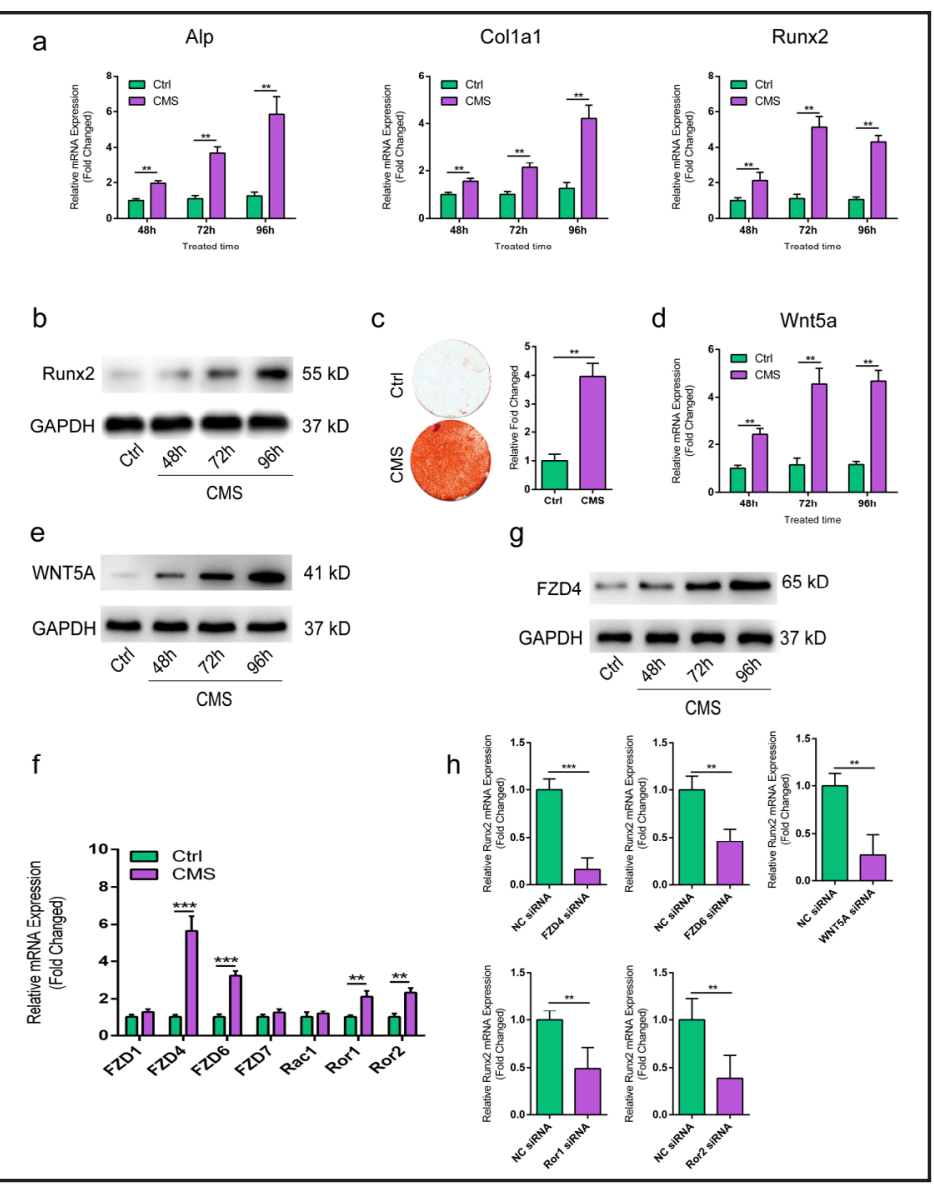
expressions of Wnt5a receptors, Frizzled (FZDs), Rac1, and the tyrosine kinase-like orphan receptors Ror1 and Ror2 were all examined by quantitative real-time PCR (qRT-PCR) after treatment with or without CMS for $48 \mathrm{~h}$. (g) Western blot analysis of the FZD4a protein expression after the application of CMS for $48 \mathrm{~h}, 72$ $\mathrm{h}$ and $96 \mathrm{~h}$. GAPDH was used as the internal control. (h) Relative Runx2 mRNA expression in BMSCs after treatment with FZD4, FZD6, WNT5A, Ror1 and Ror2 siRNA. All the data were confirmed in three repeated tests. Data are the mean \pm S.D. ${ }^{* *} \mathrm{P}<0.01,{ }^{* * *} \mathrm{P}<0.01$. All P-values are based on Student's t-test.

FZD4 shRNA treatment (Fig. 3d). In addition, to confirm the specific effects of FZD4 on the osteogenic differentiation of MSCs, we added exogenous Wnt5a protein to MSCs (Fig. 3e) with or without FZD4 shRNA treatment. We found that Wnt5a protein promoted the CMSinduced JNK activation and osteogenic differentiation of BMSCs, as shown by the increased expression of phosphorylated JNK and the ALP staining (Fig. 3f and 3g). However, when we inhibited the FZD4 expression with shRNA, the CMS-Wnt5a-activated JNK signalling, enhanced expression of the osteogenic markers Col1a1, Alp and Runx2, and osteogenic differentiation were abrogated significantly, as evidenced by the western blot results and ALP staining (Fig. 3h-i). These results confirmed that the FZD4 receptor mediated the CMSWnt5a-induced osteogenic differentiation of BMSCs.

\section{Overexpression of Wnt5a/FZD4 rescued the decrease in bone formation in vivo}

To investigate the function of FZD4 in vivo, we used the HU mouse model. We used microCT to measure the bone density, and the results showed that the mechanical unloadinginduced bone loss was partly rescued by Wnt5a addition, as shown by microCT (Fig. 4a). Bone volume analysis revealed that the bone volume-related parameters, such as BMD, BV/TV and $\mathrm{Tb}$.Th, were partly increased by the Wnt5a addition (Fig. 4b). However, the bone formation 
Fig. 3. FZD4 is indispensable for the CMS-induced osteogenic differentiation of BMSCs. (a) qRTPCR analysis and (b) Western blot analysis of FZD4 in BMSCs after infection with lentiviral-scramshRNA or lentiviral-FZD4-shRNA. GAPDH was used as the internal control. (c) Western blot analysis of the P-JNK1, t-JNK1, and Runx2 expression in BMSCs infected with lentiviral-scram-shRNA or lentiviral-FZD4-shRNA after the application of CMS. GAPDH was used as the internal control. (d) ALP staining analysis of the ALP activity in BMSCs infected with lentiviral-scram-shRNA or lentiviral-FZD4-shRNA after the application of CMS. (e) Western blot analysis of WNT5A in BMSCs after infected with lentiviral-Vector or lentiviral-WNT5A. GAPDH was used as the internal control. (f) Western blot analysis of the P-JNK1, t-JNK1, Runx2 expression in BMSCs infected with lentiviral-Vector or lentiviral-WNT5A after the application of CMS. GAPDH was used as the internal control. (g) ALP staining analysis of the ALP activity in BMSCs infected with lentiviral-Vector or lentiviral-WNT5A after the application of CMS. (h) Western blot analysis of P-JNK1, t-JNK1, Runx2 expression in BMSCs infected with lentiviral-FZD4-shRNA and lentiviral-WNT5A after the application of CMS. GAPDH was used as the internal control. (i) ALP staining analysis of the ALP activity in BMSCs infected with lentiviral-FZD4-shRNA and lentiviral-WNT5A after the application of CMS. All the data were confirmed in three repeated tests. Data are mean \pm S.D. ${ }^{* *} \mathrm{P}<0.01$. All P-values are based on Student's t-test.

ability in vivo was obviously increased in the FZD4 overexpression plus Wnt5a added group, as confirmed by microCT, calcein and Alizarin red labelling and the bone formation-related parameter MAR (Fig. 4a, 4b, 4d and 4f). On the other hand, the bone resorption ability in vivo was obviously decreased in the FZD4 overexpression plus Wnt5a added group, as confirmed by TRAP staining (Fig. 4c and 4e). Then, we overexpressed FZD4 in isolated BMSCs from normal and HU mice, and we demonstrated that the HU-impaired JNK signalling, decreased expression of the osteogenic marker Runx2, and osteogenic differentiation were rescued significantly by FZD4 overexpression plus Wnt5a addition, as evidenced by the western blot results and ALP staining (Fig. 4g-i).

Taken together, these results indicate that FZD4 is indispensable for the CMS-induced osteogenic differentiation of BMSCs by, at least in part, mediating CMS-Wnt5a signalling and activating the downstream JNK pathway. The therapeutic addition of FZD4 and Wnt5a (by shRNA or an inhibitor of its methyltransferase activity) was able to partly counteract the bone loss observed in osteoporosis.

\section{Discussion}

Wht signalling plays essential roles in the development and homeostasis of the skeletal system. Wnt5a-activated signalling has been demonstrated to suppress adipogenesis, which in turn promoted the osteogenic differentiation of MSCs [21]. Previous studies have shown that various mechanical stimuli enhanced the expression of Wnt5a mRNA in KARGER 


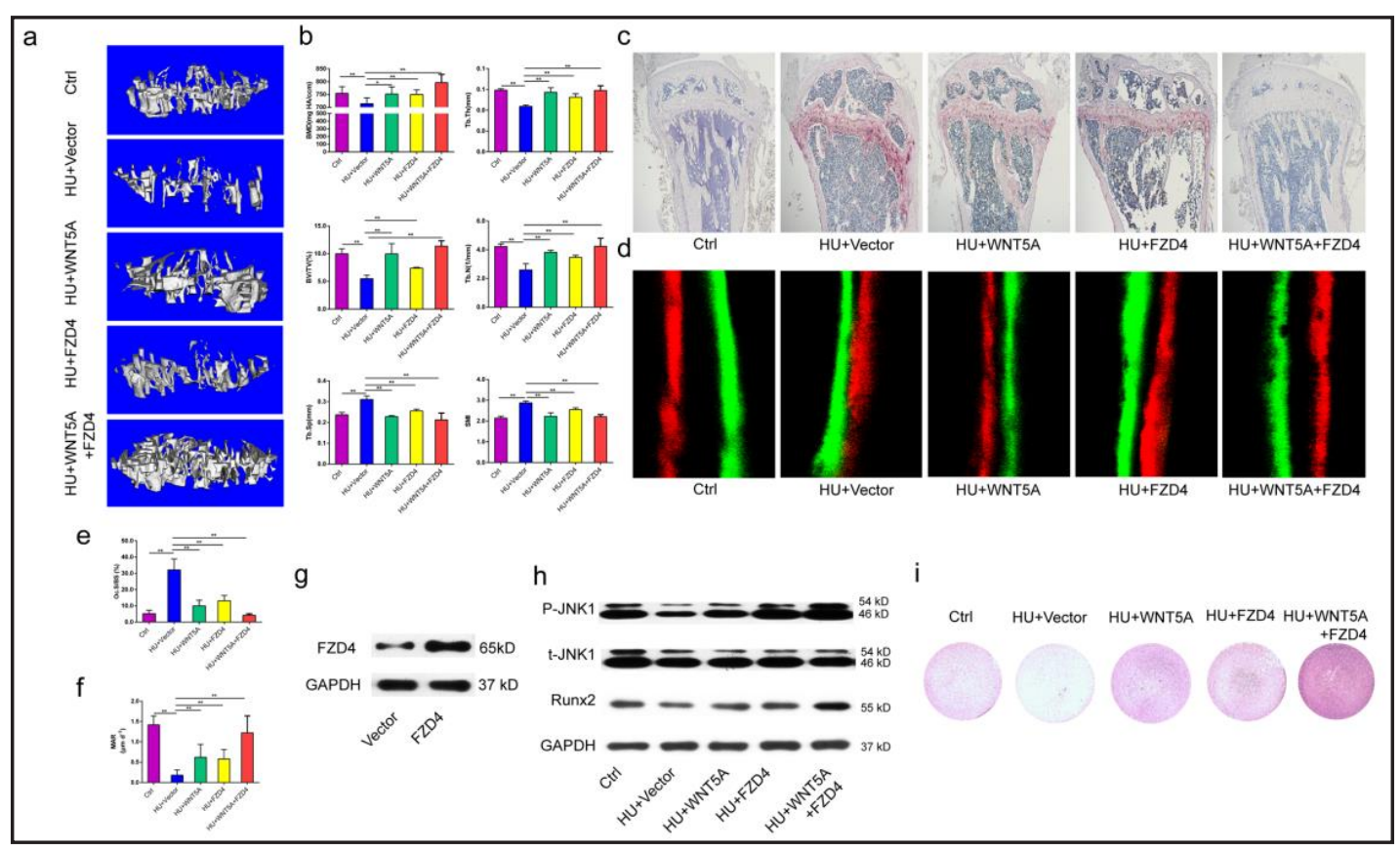

Fig. 4. Overexpression of Wnt5a/FZD4 rescued the decrease in bone formation in vivo. (a) Representative images of microCT reconstruction images of the tibial plateau from each group. $n=6$. (b) 3D structural parameters-BMD, BV/TV, Tb.N, Tb.Sp, Tb.Th and SMI-of the tibial plateau determined by microCT in each group. $n=6$. (c) Representative images of decalcified bone stained with TRAP in each group. Magnification 40×. (d) Representative images showing new bone formation assessed by Alizarin red and calcein labelling in each group. (e) Quantitative analyses of osteoclast surface/bone surface (Oc.S/BS). n=6. (f) Histomorphometric analysis of the bone formation-related parameter MAR in each group. $\mathrm{n}=6$. Magnification 200×. (g) Western blot analysis of the FZD4 protein expression in BMSCs infected with lentiviral-vector or lentiviral-FZD4. GAPDH was used as the internal control. (h) Western blot analysis of the P-JNK1, t-JNK1, and Runx2 expression in BMSCs infected with lentiviral-FZD4 and lentiviral-WNT5A after the application of CMS. GAPDH was used as the internal control. (i) ALP staining analysis of the ALP activity in BMSCs infected with lentiviral-FZD4 and lentiviral-WNT5A after the application of CMS. All the data were confirmed in three repeated tests. Data are the mean \pm S.D. ${ }^{* *} \mathrm{P}<0.01$. All P-values are based on Student's t-test.

stem cells and therefore promoted osteogenic differentiation [22, 23]_ENREF_10. Wnt5a mediated the fluid shear stress-induced osteogenic differentiation of C3H10T1/2 cells by inducing $\beta$-catenin translocation. When Wnt5a was inhibited by siRNA, the osteogenic differentiation of C3H10T1/2 cells was blocked. In tendon-derived stem cells, Wnt5a, but not other noncanonical Wnts, was upregulated after uniaxial mechanical tension treatment. The knockdown of Wnt5a expression decreased the Runx 2 expression induced by uniaxial mechanical tension [24, 25]. Consistent with these previous reports, we compared the expression profiles of BMSCs from osteoporotic patients with those of normal people, and we found that the expression of osteogenic differentiation markers in BMSCs was decreased, while the expression of Wnt5a and FZD4 was downregulated, which indicated that osteogenic differentiation of BMSCs may be controlled by Wnt5a/FZD 4 signalling. Next, we showed that CMS promoted the expression of Wnt5a and the osteogenic differentiation of BMSCs. The addition of exogenous Wnt5a protein promoted the CMS-induced JNK and Runx2 expression and the osteogenic differentiation of BMSCs in vitro and in vivo. These data demonstrated that CMS could enhance the expression of Wnt5a mRNA in BMSCs and autonomously promoted osteogenic differentiation.

The role of Wnt5a receptors in the mechanical stress-induced osteogenic differentiation of BMSCs has not been deeply investigated. Previous studies showed that Wnt5a activated noncanonical Wnt signalling by binding the receptor Ror2 and activating the JNK signalling

\section{KARGER}


pathway [26, 27]. However, Ror2 knockout mice did not have impaired bone formation. Wnt5a was also shown to bind a receptor complex of FZD4 and Lrp5 and activate Wnt/ $\beta$ catenin signalling in HEK293 cells [28]. Wnt5a by itself induced noncanonical signalling and upregulated Lrp5/6 expression in osteoblast-lineage cells to increase their sensitivity to canonical Wnt ligands [16]. However, the role of the Wnt5a receptor FZD4 under mechanical stress conditions has not been clarified. In our study, we examined the roles of FZD4 in osteogenic differentiation. We performed gene-silencing experiments by shRNA. When shRNAs were used to knockdown the expression of FZD4, the CMS-induced osteogenic differentiation of BMSCs was inhibited, indicating that the CMS-induced osteogenic differentiation of BMSCs was regulated by FZD4. Knockdown of FZD4 decreased the CMSinduced expression of Runx2, which is a marker of the early of osteogenic differentiation and the downstream JNK signalling. Overexpression of FZD4 increased the CMS-induced ALP activity, the expression of Runx2 and the osteogenic differentiation. Overexpression of FZD4 also partly rescued the HU-induced impaired osteogenic differentiation of BMSCs. To further clarify the role of FZD4 in bone formation in vivo, we used the hindlimb unloading mouse model and found that FZD4 overexpression rescued the bone formation of BMSCs. Taken together, this information indicates that FZD4 promoted the CMS-induced osteogenic differentiation and bone formation.

\section{Conclusion}

In summary, our study provides new findings that mechanical stimulation orchestrates gene expression for the osteogenic differentiation of BMSCs by directly regulating the WNT5A/FZD4/JNK signalling pathway. FZD4 functions through activated JNK, which is the master regulator of osteogenesis. Understanding the molecular mechanisms of the WNT5A/ FZD4/JNK signalling pathway in regulating MSC lineage determination under mechanical stimulation is pivotal for understanding bone cell differentiation and diseases. These findings not only provide new insights into mechanoresponse signalling pathways but also raise intriguing possibilities for the use of FZD4 modulators to regulate bone formation in regenerative medicine. We anticipate that our study will provide a foundation for future investigations on the development of gene therapies for treating human bone remodelling disorders related to mechanical loading, such as osteoporosis.

\section{Acknowledgements}

This work was supported by grants from the National Natural Science Foundation of China (No. 81572168) and the Science and Technology Commission of Shanghai Municipality (No. 15411950700).

\section{Disclosure Statement}

The authors declare that the research was conducted in the absence of any commercial or financial relationships that could be construed as a potential conflict of interests.

\section{References}

1 Hu H, Chen M, Dai G, Du G, Wang X, He J, Zhao Y, Han D, Cao Y, Zheng Y, Ding D: An inhibitory role of osthole in rat mscs osteogenic differentiation and proliferation via wnt/beta-catenin and erk1/2-mapk pathways. Cell Physiol Biochem 2016;38:2375-2388. 


\section{Cellular Physiology Cell Physiol Biochem 2018;48:215-226 \begin{tabular}{l|l} 
DOI: 10.1159/000491721 & $\begin{array}{l}\text { O } 2018 \text { The Author(s). Published by S. Karger AG, Basel } \\
\text { www.karger.com/cpb }\end{array}$
\end{tabular}}

Gu et al.: Wnt5a Mediates Mechanical Stretch Induced Osteogenesis

-2 Justesen J, Stenderup K, Ebbesen EN, Mosekilde L, Steiniche T, Kassem M: Adipocyte tissue volume in bone marrow is increased with aging and in patients with osteoporosis. Biogerontology 2001;2:165-171.

3 Song F, Sun H, Huang L, Fu D, Huang C: The role of pannexin3-modified human dental pulp-derived mesenchymal stromal cells in repairing rat cranial critical-sized bone defects. Cell Physiol Biochem 2017;44:2174-2188.

4 Chen YX, Zhu DY, Xu ZL, Yin JH, Yu XW, Mei J, Gao YS, Zhang CQ: The protective effect of cordycepin on alcohol-induced osteonecrosis of the femoral head. Cell Physiol Biochem 2017;42:2391-2403.

$>5$ Rittweger J, Frost HM, Schiessl H, Ohshima H, Alkner B, Tesch P, Felsenberg D: Muscle atrophy and bone loss after 90 days' bed rest and the effects of flywheel resistive exercise and pamidronate: Results from the ltbr study. Bone 2005;36:1019-1029.

6 Zwart SR, Pierson D, Mehta S, Gonda S, Smith SM: Capacity of omega-3 fatty acids or eicosapentaenoic acid to counteract weightlessness-induced bone loss by inhibiting nf-kappab activation: From cells to bed rest to astronauts. J Bone Miner Res 2010;25:1049-1057.

7 Kelly DJ, Jacobs CR: The role of mechanical signals in regulating chondrogenesis and osteogenesis of mesenchymal stem cells. Birth Defects Res C Embryo Today 2010;90:75-85.

8 Angele P, Yoo JU, Smith C, Mansour J, Jepsen KJ, Nerlich M, Johnstone B: Cyclic hydrostatic pressure enhances the chondrogenic phenotype of human mesenchymal progenitor cells differentiated in vitro. J Orthop Res 2003;21:451-457.

-9 Chen JC, Jacobs CR: Mechanically induced osteogenic lineage commitment of stem cells. Stem Cell Res Ther 2013;4:107.

10 Wang J, Qin Y, Mi X: The protective effects of bone marrow-derived mesenchymal stem cell (bmsc) on lps-induced acute lung injury via tlr3-mediated ifns, mapk and nf-kappab signaling pathways. Biomed Pharmacother 2016;79:176-187.

11 Tang Z, Wei J, Yu Y, Zhang J, Liu L, Tang W, Long J, Zheng X, Jing W: Gamma-secretase inhibitor reverts the notch signaling attenuation of osteogenic differentiation in aged bone marrow mesenchymal stem cells. Cell Biol Int 2016;40:439-447.

12 Wang Y, Zhang X, Shao J, Liu H, Liu X, Luo E: Adiponectin regulates bmsc osteogenic differentiation and osteogenesis through the wnt/beta-catenin pathway. Sci Rep 2017;7:3652.

13 Kong X, Li X, Zhang C, Zhu L, Liu C, Qin Q Liu C, Wang Q, Zhu J, Wu X, Wan H, Chen W, Lin N: Ethyl acetate fraction of huogu formula inhibits adipogenic differentiation of bone marrow stromal cells via the bmp and wnt signaling pathways. Int J Biol Sci 2017;13:480-491.

14 Takada I, Kouzmenko AP, Kato S: Ppar-gamma signaling crosstalk in mesenchymal stem cells. PPAR Res 2010;2010.

15 Takada I, Suzawa M, Matsumoto K, Kato S: Suppression of ppar transactivation switches cell fate of bone marrow stem cells from adipocytes into osteoblasts. Ann N Y Acad Sci 2007;1116:182-195.

16 Okamoto M, Udagawa N, Uehara S, Maeda K, Yamashita T, Nakamichi Y, Kato H, Saito N, Minami Y, Takahashi N, Kobayashi Y: Noncanonical wnt5a enhances wnt/beta-catenin signaling during osteoblastogenesis. Sci Rep 2014;4:4493.

17 Breton-Romero R, Feng B, Holbrook M, Farb MG, Fetterman JL, Linder EA, Berk BD, Masaki N, Weisbrod RM, Inagaki E, Gokce N, Fuster JJ, Walsh K, Hamburg NM: Endothelial dysfunction in human diabetes is mediated by wnt5a-jnk signaling. Arterioscler Thromb Vasc Biol 2016;36:561-569.

18 Feng Y, Su L, Zhong X, Guohong W, Xiao H, Li Y, Xiu L: Exendin-4 promotes proliferation and differentiation of mc3t3-e1 osteoblasts by mapks activation. J Mol Endocrinol 2016;56:189-199.

19 Subramanian A, Tamayo P, Mootha VK, Mukherjee S, Ebert BL, Gillette MA, Paulovich A, Pomeroy SL, Golub TR, Lander ES, Mesirov JP: Gene set enrichment analysis: A knowledge-based approach for interpreting genome-wide expression profiles. Proc Natl Acad Sci U S A 2005;102:15545-15550.

20 Wang J, Wang CD, Zhang N, Tong WX, Zhang YF, Shan SZ, Zhang XL, Li QF: Mechanical stimulation orchestrates the osteogenic differentiation of human bone marrow stromal cells by regulating hdac1 Cell Death Dis 2016; 7:e2221.

21 Krishnan V, Bryant HU, Macdougald OA: Regulation of bone mass by wnt signaling. J Clin Invest 2006;116:1202-1209.

22 Charoenpanich A, Wall ME, Tucker CJ, Andrews DM, Lalush DS, Dirschl DR, Loboa EG: Cyclic tensile strain enhances osteogenesis and angiogenesis in mesenchymal stem cells from osteoporotic donors. Tissue Eng Part A 2014;20:67-78. 


\section{Cellular Physiology Cell Physiol Biochem 2018;48:215-226 \begin{tabular}{l|l} 
DOI: 10.1159/000491721 & O 2018 The Author(s). Published by S. Karger AG, Basel \\
www.karger.com/cpb
\end{tabular} \\ Gu et al.: Wnt5a Mediates Mechanical Stretch Induced Osteogenesis}

-23 Johnson ML, Kamel MA: The wnt signaling pathway and bone metabolism. Curr Opin Rheumatol 2007;19:376-382.

-24 Liu X, Chen W, Zhou Y, Tang K, Zhang J: Mechanical tension promotes the osteogenic differentiation of rat tendon-derived stem cells through the wnt5a/wnt5b/jnk signaling pathway. Cell Physiol Biochem 2015;36:517-530.

25 Shi Y, Fu Y, Tong W, Geng Y, Lui PP, Tang T, Zhang X, Dai K: Uniaxial mechanical tension promoted osteogenic differentiation of rat tendon-derived stem cells (rtdscs) via the wnt5a-rhoa pathway. J Cell Biochem 2012;113:3133-3142.

26 Maeda K, Kobayashi Y, Udagawa N, Uehara S, Ishihara A, Mizoguchi T, Kikuchi Y, Takada I, Kato S, Kani S, Nishita M, Marumo K, Martin TJ, Minami Y, Takahashi N: Wnt5a-ror2 signaling between osteoblast-lineage cells and osteoclast precursors enhances osteoclastogenesis. Nat Med 2012;18:405-412.

27 Oishi I, Suzuki H, Onishi N, Takada R, Kani S, Ohkawara B, Koshida I, Suzuki K, Yamada G, Schwabe GC, Mundlos S, Shibuya H, Takada S, Minami Y: The receptor tyrosine kinase ror2 is involved in non-canonical wnt5a/jnk signalling pathway. Genes Cells 2003;8:645-654.

-28 Mikels AJ, Nusse R: Purified wnt5a protein activates or inhibits beta-catenin-tcf signaling depending on receptor context. PLoS Biol 2006;4:e115. 